\title{
Idiopathic Dural Optic Nerve Sheath Calcification Associated with Sclerochoroidal Calcification: Case Report and Review of Literatures
}

\author{
Mohammad Sharifi $^{a} \quad$ Bahar Tafaghodi Yousefi $^{b}$ \\ ${ }^{a}$ Associate Professor of Ophthalmology, Eye Research Center, Mashhad University of \\ Medical Science, Mashhad, Iran; bellowship in Strabismus, Mashhad University of Medical \\ Science, Mashhad, Iran
}

\section{Keywords}

Dural sheath $\cdot$ Optic nerve $\cdot$ Calcification $\cdot$ Sclera $\cdot$ Choroid

\begin{abstract}
We present a patient with idiopathic intracranial calcifications with simultaneous involvement of sclera, choroid, optic nerve, and trochlear apparatus. A 70-year-old woman with bilateral decreased vision was referred to our hospital. Ocular examinations revealed sclerochoroidal mass beneath superotemporal vascular arcade in macular area. Orbital CT scan shows bilateral calcification of dural optic nerve sheath and posterior wall of the globe as well as calcification in the trochlear apparatus and brain. Ultrasound showed highly reflective echogenic lesion with shadowing. OCT revealed rolling topography with thinning of the overlying choroid and outer nuclear layer as well as absence of the external limiting membrane and inner segment-outer segment junction. Slight irregular thickening of the retinal pigment epithelium is present. It is the first case of simultaneous bilateral dural optic nerve sheath and sclerochoroidal calcification.
\end{abstract}

\section{Introduction}

Idiopathic intracranial calcification involves dura of the falx cerebri, tentorium cerebellum, or calvaria. Coexistence of optic nerve dural sheath, sclera, and trochlear apparatus of superior oblique calcifications have been reported [1]. Sclerochoroidal calcification (SCC) is a rare incidental finding in fundoscopy. Clinical appearance of SCC simulates intraocular tumors such as choroidal metastasis, choroidal melanoma, choroidal osteoma, and 
melanoma [2]. Since there are many differential diagnoses for SCC and dural optic nerve calcification based on clinical appearance, further evaluation should be done. Specific imaging features such as sonography, CT scan, or OCT can help to differentiate these entities. Calcifications may be associated systemic diseases such as hyperparathyroidism, pseudo hypoparathyroidism, parathyroid adenoma, Gitelman syndrome, and Bartter syndrome [3]. We present a case of idiopathic calcification involving dural optic nerve sheath, sclera, choroid trochlea, and brain.

\section{Case Report}

A 70-year-old woman with gradually bilateral decreased vision was referred to our eye hospital. Best-corrected visual acuity was $0.50 \log$ MAR in right eye and 1.0 log MAR in left eye. She had normal motility and alignment with no proptosis. Anterior segment examination revealed aphakia of both eyes secondary to previous cataract surgery. Fundus examination of the right eye was unremarkable. In the left eye, there was a dome-shaped orange mass beneath a superotemporal vascular arcade in macular area. Orbital CT scan shows bilateral calcification of dural optic nerve sheath and posterior wall of the globe as well as calcification in the trochlear apparatus and brain (Fig. 1). Ultrasound showed highly reflective echogenic lesion with shadowing (Fig. 2). On OCT, the mass was depicted within the sclera, showing marked elevation that pushed choroid and retina inward associated with thinning of outer nuclear layer as well as absence of the external limiting membrane and inner segment-outer segment junction. There was no subretinal fluid (Fig. 3). She was referred to an endocrinologist to rule out systemic conditions. All metabolic evaluation was normal.

Fig. 1. Orbital CT scan shows bilateral calcification of dural optic nerve sheath and posterior wall of the globe as well as calcification in the trochlear apparatus and brain.
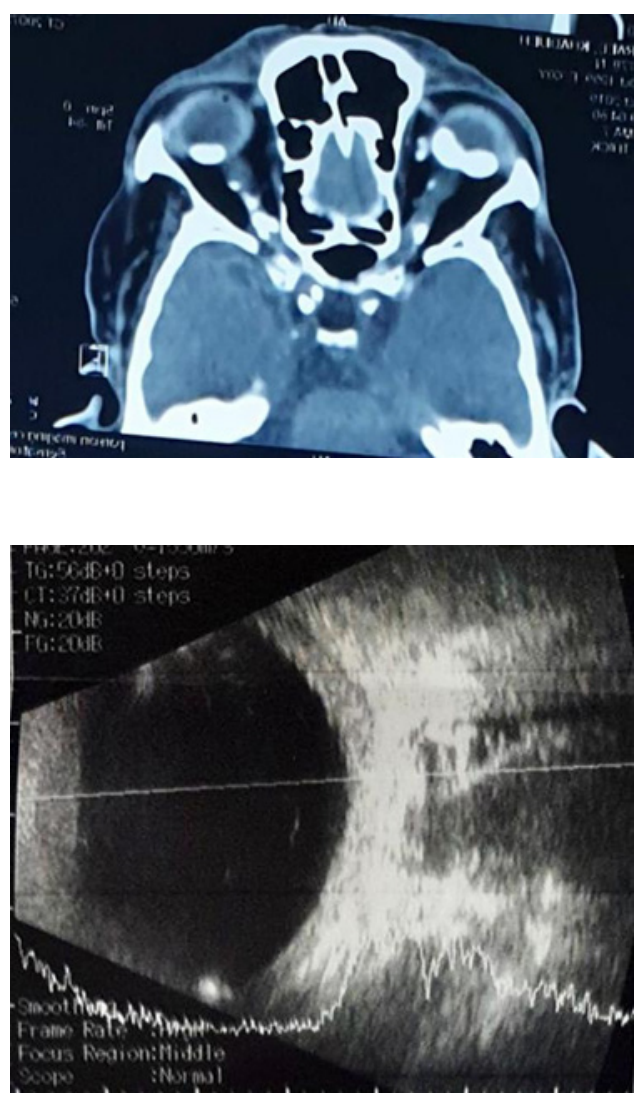

sion with shadowing. 

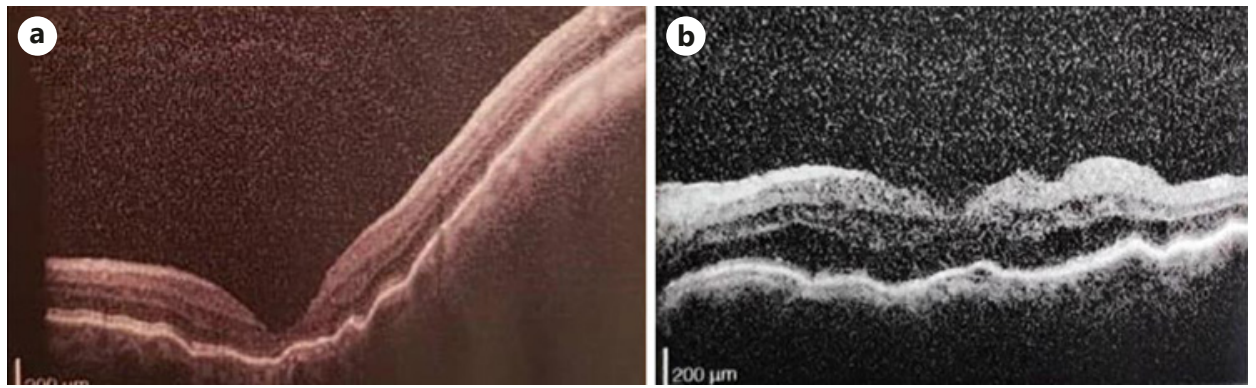

Fig. 3. On EDI-OCT (vertical (a) and horizontal (b) scan), the mass was depicted within the sclera, showing marked elevation that pushed the choroid and retina inward with thinning of the overlying choroid and outer nuclear layer as well as absence of the external limiting membrane and inner segment-outer segment junction. There was no subretinal fluid.

\section{Discussion}

Calcium salt deposition occurs in 2 pathologic forms, ossification as bone structure tissues change and calcification as an amorphous calcium deposition. Metastatic calcification is defined as the deposition of calcium salts in normal tissue due to calcium and phosphate metabolism abnormalities such as hyperparathyroidism, pseudo hypoparathyroidism, and renal dysfunction. Dystrophic is the calcium deposition in abnormal tissue with normal serum calcium and phosphate salts. It is a degenerative process as a result of aging, trauma, and chronic inflammation [2]. Systemic treatable pathologies should be ruled out by biochemical tests [4]. These include serum calcium, phosphorus, potassium, magnesium, vitamin D, serum hormones calcitonin, thyroid-stimulating hormone, and parathyroid hormone [5]. The most common associated systemic conditions include hyperparathyroidism, parathyroid adenoma, Bartter syndrome, and Gitelman syndrome [3]. Because we ruled out systemic causes of calcification and did not find any cause, she had idiopathic dural optic nerve sheath and SCC. Benign calcifications of the dura of the falx cerebri, tentorium cerebellum, or calvaria have been found in ten percent of normal individuals. These intracranial calcification have been reported in company with the optic nerve head, trochlear apparatus of the superior oblique muscle, the sclera, and proximal dural sheaths of the optic nerve calcifications [1]. Nicholson et al. [6] reported the case of a 58-year-old man who was incidentally found to have bilateral optic nerve calcification with extensive intracranial dural calcification simultaneously. This patient had no other comorbidities that could explain the calcifications and so a diagnosis of idiopathic dural optic nerve sheath calcification was made [6]. None of the dural optic nerve sheath calcifications have been reported in literatures accompany with SCC as in our case. Murray et al. [1] reported the incidence of $2 \%$ bilateral drusen of the optic nerve head, $3 \%$ calcified scleral plaques anterior to the medial or lateral rectus muscles, and $3 \%$ bilateral ossification of the trochlear apparatus in 100 orbital CT scans. SCC has been recognized as dome shape or plaque mass in asymptomatic old-age individuals. The SCC is usually bilateral and located along superior and inferior vascular arcade [7]. Fung et al. [7] analyzed 17 SCC patients based on EDI-OCT and suggested that SCCs were in sclera associated with choroidal compression or absence. The authors describe the irregular surface contour of SCC as "rocky and rolling" configuration. The rocky topography showed marked pointed elevation and rolling showed smooth undulation of the gentle elevation [7]. Lesions often do not cause any visual disturbance because of their extra macular location [8]. Mitamura et al. [9] detected broad choroidal hypoperfusion in an ICG of SCC patient that led to impaired 
oxygenation of outer retina and RPE at macula. Laser speckle flowgraphy confirmed low choroidal blood flow presented with cold color pattern [9]. It is hypothesized that compromised choroidal vessels circulation due to calcification may cause outer retinal layers atrophy $[7,9]$. In the presented case, EDI-OCT revealed rolling topography with thinning of the overlying choroid and outer nuclear layer as well as absence of the external limiting membrane and inner segment-outer segment junction. Slight irregular thickening of the retinal pigment epithelium is present. Multimodal imaging, specially EDI-OCT, can assist to establish differential diagnosis such as choroidal osteoma, metastasis, amelanotic choroidal nevus or melanoma, lymphoma, and granuloma $[8,10,11]$. In conclusion, this is first case of idiopathic bilateral dural optic nerve sheath calcification associated with sclerochoraidal calcification which had trochlear apparatus and brain involvements.

\section{Acknowledgements}

I would like to thank everyone who played a role in this manuscript, especially my colleagues in Khatam Eye Hospital.

\section{Statement of Ethics}

This manuscript was approved by Ethics Committee of Mashhad University of Medical Sciences. Written informed consent for publication was obtained from the patient for publication of this case report and any accompanying images.

\section{Conflict of Interest Statement}

No conflict of interest.

\section{Funding Sources}

Eye Research Center, Mashhad University of Medical Sciences.

\section{Author Contributions}

Data collection, writing, and submission of this manuscript were done by Dr. Sharifi and Dr. Tafaghodi.

\section{References}

1 Murray JL, Hayman LA, Tang RA, Schiffman JS. Incidental asymptomatic orbital calcifications. J Neuroophthalmol. 1995;15(4):203-8.

2 Kumar V, Abbas AK, Aster JC, Perkins JA. Robbins basic pathology. 10th ed. Philadelphia, PA: Elsevier; 2018.

3 Shields CL, Hasanreisoglu M, Saktanasate J, Shields PW, Seibel I, Shields JA. Sclerochoroidal calcification: clinical features, outcomes, and relationship with hypercalcemia and parathyroid adenoma in 179 eyes. Retina. 2015;35(3):547-54.

4 Sierra-Rodriguez MA, Bailez Fidalgo C, Saenz-Frances F, Gonzalez Romero JC, Munoz Bellido L. Sclerochoroidal calcification associated with hypovitaminosis D. Arch Soc Esp Oftalmol. 2014;89:290-2.

\section{Karger's}


5 Hundle R, Turaka K, Shields CL. Sclerochoroidal calcification resembling choroidal metastasis. Retin Today. 2011;(July/August):57-8.

6 Nicholson BP, Lystad LD, Emch TM, Singh AD. Idiopathic dural optic nerve sheath calcification. Br J Ophthalmol. 2011;95(2):290-9.

7 Fung AT, Arias JD, Shields CL, Shields JA. Sclerochoroidal calcification is primarily a scleral condition based on enhanced depth imaging optical coherence tomography. JAMA Ophthalmol. 2013;131(7):960-3.

8 Honavar SG, Shields CL, Demirci H, Shields JA. Sclerochoroidal calcification: clinical manifestations and systemic associations. Arch Ophthalmol. 2001;119(6):833-40.

9 Mitamura M, Kase S, Ishida S. Multimodal imaging in sclerochoroidal calcification: a case report and literature review. BMC Ophthalmol. 2020;20(1):248.

10 Nicholson BP, Lystad LD, Emch TM, Singh AD. Idiopathic dural optic nerve sheath calcification. Br J Ophthalmol. 2008;95(2):290-9.

11 Ascaso FJ, Lasierra R. Idiopathic dural optic nerve sheath calcification associated with choroidal osteoma. Ophthalmic Surg Lasers Imaging. 2011;9(42):53-5. 